A Nonlinear ParaExp Algorithm

Gander, Martin J. and Güttel, Stefan and Petcu, Madalina

2017

MIMS EPrint: 2017.17

Manchester Institute for Mathematical Sciences

School of Mathematics

The University of Manchester

\footnotetext{
Reports available from: http://eprints.maths.manchester.ac.uk/

And by contacting: The MIMS Secretary

School of Mathematics

The University of Manchester

Manchester, M13 9PL, UK
} 


\section{A Nonlinear ParaExp Algorithm}

Martin J. Gander, Stefan Güttel, and Madalina Petcu

\section{Derivation of the Nonlinear ParaExp Algorithm}

Time parallelization has a long history, see [1] and references therein. The parallel speedup obtained is in general not as good as with space parallelization, especially for hyperbolic problems. A notable exception are waveform relaxation-type methods $[3,4]$, which in the hyperbolic case are related to the more recent tent-pitching approach [6], and the ParaExp algorithm [7, 9] based on Krylov methods, which is however restricted to linear problems. For an application in a nonlinear context, see [10], and for a different approach using Krylov information, see [8]. Here we propose and analyze a variant of the ParaExp algorithm for the nonlinear initial value problem

$$
\mathbf{u}^{\prime}(t)=A \mathbf{u}(t)+B(\mathbf{u}(t))+\mathbf{g}(t), \quad t \in[0, T], \quad \mathbf{u}(0)=\mathbf{u}_{0},
$$

with $A \in \mathbb{C}^{m \times m}, B: \mathbb{C}^{m} \rightarrow \mathbb{C}^{m}$ a nonlinear operator, $\mathbf{g}:[0, T] \rightarrow \mathbb{C}^{m}$ a source function, and $\mathbf{u}:[0, T] \rightarrow \mathbb{C}^{m}$ the sought solution. Throughout this note we assume that all stated initial value problems have unique solutions. For the ParaExp algorithm, the time interval $[0, T]$ is partitioned into $N$ subintervals $\left[T_{n-1}, T_{n}\right]$ with $n=1, \ldots, N$, and a direct application of this algorithm to the nonlinear problem (1.1) gives

Step 1: Solve for $n \geq 1$ in parallel the nonlinear problems with zero initial data

$$
\begin{aligned}
\mathbf{v}_{n}^{\prime}(t) & =A \mathbf{v}_{n}(t)+B\left(\mathbf{v}_{n}(t)\right)+\mathbf{g}(t), \quad t \in\left[T_{n-1}, T_{n}\right], \\
\mathbf{v}_{n}\left(T_{n-1}\right) & =\mathbf{0} .
\end{aligned}
$$

M. J. Gander

Université de Genève, 2-4 rue du Lièvre, 1211 Genève, e-mail: Martin.Gander@unige.ch

S. Güttel

School of Mathematics, The University of Manchester, United Kingdom,

e-mail: stefan.guettel@ manchester.ac.uk

M. Petcu

Laboratoire de Mathématiques, Université de Poitiers, France,

e-mail: Madalina.Petcu@math.univ-poitiers.fr

The Institute of Mathematics of the Romanian Academy, Bucharest, Romania 
Step 2: Solve for $n \geq 1$ in parallel the linear non-homogeneous problems

$$
\begin{aligned}
\mathbf{w}_{n}^{\prime}(t) & =A \mathbf{w}_{n}(t), & & t \in\left[T_{n-1}, T\right], \\
\mathbf{w}_{n}\left(T_{n-1}\right) & =\mathbf{v}_{n-1}\left(T_{n-1}\right), & & \mathbf{v}_{0}\left(T_{0}\right)=\mathbf{u}_{0} .
\end{aligned}
$$

ParaExp then forms the linear combination $\mathbf{u}(t)=\mathbf{v}_{n}(t)+\sum_{j=1}^{n} \mathbf{w}_{j}(t), t \in\left[T_{n-1}, T_{n}\right)$, which still satisfies the initial condition, but not equation (1.1) since $\mathbf{u}^{\prime}(t)=A \mathbf{u}(t)+$ $B\left(\mathbf{v}_{n}(t)\right)+\mathbf{g}(t), t \in\left[T_{n-1}, T_{n}\right]$, except when $B$ is not present. One can however naturally separate the solution into $\mathbf{u}(t)=\mathbf{v}(t)+\mathbf{w}(t)$, with $\mathbf{w}$ solving the linear problem $\mathbf{w}^{\prime}(t)=A \mathbf{w}(t), \mathbf{w}(t)=\mathbf{u}_{0}$, and $\mathbf{v}$ solving the nonlinear remaining part $\mathbf{v}^{\prime}(t)=A \mathbf{v}(t)+B(\mathbf{v}(t)+\mathbf{w}(t))+\mathbf{g}(t), \mathbf{v}(0)=\mathbf{0}$. To apply this splitting on multiple time intervals $\left[T_{n-1}, T_{n}\right]$ we need to iterate. Using the initialization $\mathbf{v}_{n}^{0}\left(T_{n}\right)=\mathbf{0}$ for $n=1, \ldots, N$ (or some other approximation), we perform for $k=1,2, \ldots$

Step 1: Solve for $n \geq 1$ in parallel the linear problems

$$
\begin{aligned}
\left(\mathbf{w}_{n}^{k}\right)^{\prime}(t) & =A \mathbf{w}_{n}^{k}(t), & & t \in\left[T_{n-1}, T\right], \\
\mathbf{w}_{n}^{k}\left(T_{n-1}\right) & =\mathbf{v}_{n-1}^{k-1}\left(T_{n-1}\right), & & \mathbf{w}_{1}^{k}\left(T_{0}\right)=\mathbf{u}_{0} .
\end{aligned}
$$

Step 2: Solve for $n \geq 1$ in parallel the nonlinear problems

$$
\begin{aligned}
\left(\mathbf{v}_{n}^{k}\right)^{\prime}(t) & =A \mathbf{v}_{n}^{k}(t)+B\left(\mathbf{v}_{n}^{k}(t)+\sum_{j=1}^{n} \mathbf{w}_{j}^{k}(t)\right)+\mathbf{g}(t), \quad t \in\left[T_{n-1}, T_{n}\right], \\
\mathbf{v}_{n}^{k}\left(T_{n-1}\right) & =\mathbf{0} .
\end{aligned}
$$

The new approximate solution is then defined by $\mathbf{u}^{k}(t)=\mathbf{v}_{n}^{k}(t)+\sum_{j=1}^{n} \mathbf{w}_{j}^{k}(t), t \in$ $\left[T_{n-1}, T_{n}\right)$, which now satisfies equation (1.1) on each time interval $\left[T_{n-1}, T_{n}\right)$, and $\mathbf{u}^{k}(0)=\mathbf{u}_{0}$. The solution of the linear part (1.2) can still be computed efficiently as in the ParaExp algorithm using Krylov techniques, but (1.3) requires the computation of $\sum_{j=1}^{n} \mathbf{w}_{j}^{k}$ on $\left[T_{n-1}, T_{n}\right]$, and thus would need the Krylov approximation of $\mathbf{w}_{j}^{k}$ on the entire interval $\left[T_{n-1}, T_{n}\right]$. To avoid this, we rewrite the algorithm in terms of $\mathbf{u}_{n}^{k}$ instead of $\mathbf{v}_{n}^{k}$, where $\mathbf{u}_{n}^{k}$ approximates $\mathbf{u}$ : starting with $\mathbf{u}_{n}^{0}\left(T_{n}\right)=\mathbf{w}_{j}^{0}\left(T_{n}\right)=\mathbf{0}$ for all $j$ and $n$, the nonlinear ParaExp algorithm performs for $k=1,2, \ldots$

Step 1: Solve for $n \geq 1$ in parallel the linear problems

$$
\begin{array}{rlrl}
\left(\mathbf{w}_{n}^{k}\right)^{\prime}(t) & =A \mathbf{w}_{n}^{k}(t), & & t \in\left[T_{n-1}, T\right], \\
\mathbf{w}_{n}^{k}\left(T_{n-1}\right) & =\mathbf{u}_{n-1}^{k-1}\left(T_{n-1}\right)-\sum_{j=1}^{n-1} \mathbf{w}_{j}^{k-1}\left(T_{n-1}\right), & \mathbf{w}_{1}^{k}\left(T_{0}\right)=\mathbf{u}_{0} .
\end{array}
$$

Step 2: Solve for $n \geq 1$ in parallel the nonlinear problems

$$
\begin{aligned}
\left(\mathbf{u}_{n}^{k}\right)^{\prime}(t) & =A \mathbf{u}_{n}^{k}(t)+B\left(\mathbf{u}_{n}^{k}(t)\right)+\mathbf{g}(t), \quad t \in\left[T_{n-1}, T_{n}\right], \\
\mathbf{u}_{n}^{k}\left(T_{n-1}\right) & =\sum_{j=1}^{n} \mathbf{w}_{j}^{k}\left(T_{n-1}\right),
\end{aligned}
$$


and form the new approximate solution as

$$
\mathbf{u}^{k}(t)=\mathbf{u}_{n}^{k}(t), \quad t \in\left[T_{n-1}, T_{n}\right)
$$

Remark 1. To avoid the computation of $\mathbf{u}_{n}^{k}$ as the solution of a nonlinear problem, one could linearize (1.5) by using in the nonlinear term $B\left(\mathbf{u}_{n}^{k-1}\right)$ instead of $B\left(\mathbf{u}_{n}^{k}\right)$, where $\mathbf{u}_{n}^{0}=\mathbf{0}$ or some other approximation of the solution. However, in what follows we focus on the fully nonlinear version, since then $\mathbf{u}^{k}$ is the solution of the nonlinear problem (1.1) on each time interval.

\section{Analysis of the Nonlinear ParaExp Algorithm}

We first show that the nonlinear ParaExp algorithm introduced in the previous section converges in a finite number of steps.

Theorem 1. The approximate solution $\mathbf{u}^{k}$ obtained at iteration $k$ and defined by (1.6) coincides with the exact solution $\mathbf{u}$ on the time interval $\left[T_{0}, T_{k}\right)$.

Proof. Since $\mathbf{w}_{1}^{k}\left(T_{0}\right)=\mathbf{u}_{0}$ for all $k=1,2, \ldots, \mathbf{w}_{1}^{k}=\mathbf{w}_{1}^{k-1}$ on the time interval $\left[T_{0}, T\right]$ for all $k=2,3, \ldots$ Next, for $k=1$ we have $\mathbf{u}^{1}(t)=\mathbf{u}_{1}^{1}(t)$ on $\left[T_{0}, T_{1}\right]$, and since $\mathbf{u}_{1}^{1}\left(T_{0}\right)=\mathbf{w}_{1}^{1}\left(T_{0}\right)=\mathbf{u}_{0}$ we get by the uniqueness of the solution of (1.5) that $\mathbf{u}_{1}^{1}$ coincides with the exact solution $\mathbf{u}$ on the time interval $\left[T_{0}, T_{1}\right]$.

We now prove by induction that for all $k=2,3 \ldots$ we have

$$
\mathbf{u}_{n}^{k}=\mathbf{u} \text { on }\left[T_{n-1}, T_{n}\right], \forall n \leq k, \quad \mathbf{w}_{n}^{k}=\mathbf{w}_{n}^{k-1} \text { on }\left[T_{n-1}, T\right], \forall n \leq k-1 .
$$

For $k=2$, we only need to prove property (2.1) for $\mathbf{u}^{2}$, since for $\mathbf{w}_{1}^{2}$ it is ensured by the fact that $\mathbf{w}_{1}^{k}=\mathbf{w}_{1}^{k-1}$ for all $k \geq 2$. The initial condition for $\mathbf{u}_{2}^{2}$ is

$$
\mathbf{u}_{2}^{2}\left(T_{1}\right)=\mathbf{w}_{1}^{2}\left(T_{1}\right)+\mathbf{w}_{2}^{2}\left(T_{1}\right)=\mathbf{w}_{1}^{2}\left(T_{1}\right)+\mathbf{u}_{1}^{1}\left(T_{1}\right)-\mathbf{w}_{1}^{1}\left(T_{1}\right)=\mathbf{u}_{1}^{1}\left(T_{1}\right)=\mathbf{u}\left(T_{1}\right),
$$

where we used the fact that $\mathbf{w}_{1}^{2}=\mathbf{w}_{1}^{1}$ and that $\mathbf{u}_{1}^{1}$ is the exact solution on the time interval $\left[T_{0}, T_{1}\right]$. Since $\mathbf{u}_{2}^{2}$ satisfies the same equation as $\mathbf{u}$ on the time interval $\left[T_{1}, T_{2}\right]$ and $\mathbf{u}_{2}^{2}\left(T_{1}\right)=\mathbf{u}\left(T_{1}\right), \mathbf{u}_{2}^{2}$ must coincide with $\mathbf{u}$ on $\left[T_{1}, T_{2}\right]$. But we also know that $\mathbf{u}_{1}^{2}\left(T_{0}\right)=\mathbf{w}_{1}^{2}\left(T_{0}\right)=\mathbf{u}_{0}$ and that $\mathbf{u}_{1}^{2}$ satisfies (1.5), which implies $\mathbf{u}_{1}^{2}=\mathbf{u}$ on $\left[T_{0}, T_{1}\right]$, and hence $\mathbf{u}^{2}$ coincides with the exact solution of (1.1) on the time interval $\left[T_{0}, T_{2}\right)$.

We now suppose that (2.1) holds for all iterations up to an arbitrarily fixed index $k$ and we prove $(2.1)$ for $k+1$. To first check that $\mathbf{w}_{n}^{k+1}=\mathbf{w}_{n}^{k}$ on $\left[T_{n-1}, T\right]$ for all $n=2,3, \ldots, k$, we compute

$$
\begin{aligned}
\mathbf{w}_{n}^{k+1}\left(T_{n-1}\right) & =\mathbf{u}_{n-1}^{k}\left(T_{n-1}\right)-\sum_{j=1}^{n-1} \mathbf{w}_{j}^{k}\left(T_{n-1}\right)=\mathbf{u}\left(T_{n-1}\right)-\sum_{j=1}^{n-1} \mathbf{w}_{j}^{k-1}\left(T_{n-1}\right) \\
& =\mathbf{u}_{n-1}^{k-1}\left(T_{n-1}\right)-\sum_{j=1}^{n-1} \mathbf{w}_{j}^{k-1}\left(T_{n-1}\right)=\mathbf{w}_{n}^{k}\left(T_{n-1}\right)
\end{aligned}
$$


where we have used the recurrence hypothesis (2.1). Since $\mathbf{w}_{n}^{k+1}$ and $\mathbf{w}_{n}^{k}$ satisfy the same equation and have the same initial condition, the result follows. We next prove that $\mathbf{u}_{n}^{k+1}=\mathbf{u}$ on $\left[T_{n-1}, T_{n}\right]$ for all $n \leq k+1$. Since we already know that $\mathbf{u}_{n}^{k+1}$ and $\mathbf{u}$ satisfy the same equation on the time interval $\left[T_{n-1}, T_{n}\right]$, we only need to check the initial condition satisfied by $\mathbf{u}_{n}^{k+1}$,

$$
\begin{aligned}
\mathbf{u}_{n}^{k+1}\left(T_{n-1}\right) & =\sum_{j=1}^{n} \mathbf{w}_{j}^{k+1}\left(T_{n-1}\right)=\sum_{j=1}^{n-1} \mathbf{w}_{j}^{k+1}\left(T_{n-1}\right)+\mathbf{u}_{n-1}^{k}\left(T_{n-1}\right)-\sum_{j=1}^{n-1} \mathbf{w}_{j}^{k}\left(T_{n-1}\right) \\
& =\mathbf{u}_{n-1}^{k}\left(T_{n-1}\right),
\end{aligned}
$$

where we used the first result we just proved for $\mathbf{w}_{n}^{k+1}$ and that $\mathbf{w}_{1}^{k+1}=\mathbf{w}_{1}^{k}$ for all $k$. Now, using the recurrence hypothesis (2.1), we know that $\mathbf{u}_{n-1}^{k}$ coincides with the exact solution of (1.1) on $\left[T_{n-2}, T_{n-1}\right]$, which implies that $\mathbf{u}_{n}^{k+1}\left(T_{n-1}\right)=\mathbf{u}\left(T_{n-1}\right)$.

We now show that the nonlinear ParaExp algorithm can be interpreted in the context of the Parareal algorithm written as a multiple shooting method (see [5, 2]). We will need the following result.

Lemma 1. Let $\left(\mathbf{u}_{n}^{k}\right)_{k, n}$ be the sequence defined by the nonlinear ParaExp algorithm (1.4)-(1.6). Defining $\widetilde{\mathbf{u}}_{n}^{0}\left(T_{n}\right)=\mathbf{0}$ and $\mathbf{C}_{n}^{0}\left(T_{n}\right)=\mathbf{0}$ for all $n \geq 0$, let $\left(\mathbf{C}_{n}^{k}\right)_{k, n}$ for all $k \geq 1$ and $n \geq 1$ be the solutions of the linear problems

$$
\begin{aligned}
\left(\mathbf{C}_{n}^{k}\right)^{\prime}(t) & =A \mathbf{C}_{n}^{k}(t), & & t \in\left[T_{n-1}, T_{n}\right], \\
\mathbf{C}_{n}^{k}\left(T_{n-1}\right) & =\mathbf{C}_{n-1}^{k}\left(T_{n-1}\right)+\widetilde{\mathbf{u}}_{n-1}^{k-1}\left(T_{n-1}\right)-\mathbf{C}_{n-1}^{k-1}\left(T_{n-1}\right), & & \mathbf{C}_{1}^{k}\left(T_{0}\right)=\mathbf{u}_{0},
\end{aligned}
$$

and let $\left(\widetilde{\mathbf{u}}_{n}^{k}\right)_{k, n}$ be the solutions of the nonlinear problems

$$
\begin{aligned}
\left(\widetilde{\mathbf{u}}_{n}^{k}\right)^{\prime}(t) & =A \widetilde{\mathbf{u}}_{n}^{k}(t)+B\left(\widetilde{\mathbf{u}}_{n}^{k}(t)\right)+\mathbf{g}(t), \quad t \in\left[T_{n-1}, T_{n}\right], \\
\widetilde{\mathbf{u}}_{n}^{k}\left(T_{n-1}\right) & =\mathbf{C}_{n}^{k}\left(T_{n-1}\right) .
\end{aligned}
$$

Then $\mathbf{u}_{n}^{k}=\widetilde{\mathbf{u}}_{n}^{k}$ on $\left[T_{n-1}, T_{n}\right]$ for all $n \geq 0$ and $k \geq 1$.

Proof. At step $k=1$ and for all $n \geq 1, \mathbf{C}_{n}^{1}$ is the solution of the linear problem

$$
\begin{aligned}
\left(\mathbf{C}_{n}^{1}\right)^{\prime}(t) & =A \mathbf{C}_{n}^{1}(t), & & t \in\left[T_{n-1}, T_{n}\right], \\
\mathbf{C}_{n}^{1}\left(T_{n-1}\right) & =\mathbf{C}_{n-1}^{1}\left(T_{n-1}\right), & & \mathbf{C}_{1}^{1}\left(T_{0}\right)=\mathbf{u}_{0} .
\end{aligned}
$$

Hence $\mathbf{C}_{n}^{1}$ is the restriction of the solution of $\mathbf{u}^{\prime}=A \mathbf{u}, \mathbf{u}(0)=\mathbf{u}_{0}$ on $\left[T_{0}, T\right]$ to the time interval $\left[T_{n-1}, T_{n}\right]$. Taking into account the definition (1.4) of $\mathbf{w}_{n}^{1}$, we notice that $\mathbf{w}_{n}^{1}=\mathbf{0}$ for $n>1$ and $\mathbf{w}_{1}^{1}$ is the solution of the linear problem $\mathbf{u}^{\prime}=A \mathbf{u}, \mathbf{u}(0)=\mathbf{u}_{0}$ on $\left[T_{0}, T\right]$. Thus, $\mathbf{C}_{n}^{1}(t)=\sum_{j=1}^{n} \mathbf{w}_{j}^{1}(t)$ on $\left[T_{n-1}, T_{n}\right]$, and $\widetilde{\mathbf{u}}_{n}^{1}$ satisfies for $n \geq 1$ 


$$
\begin{aligned}
\left(\widetilde{\mathbf{u}}_{n}^{1}\right)^{\prime}(t) & =A \widetilde{\mathbf{u}}_{n}^{1}(t)+B\left(\widetilde{\mathbf{u}}_{n}^{1}(t)\right)+\mathbf{g}(t), \quad t \in\left[T_{n-1}, T_{n}\right], \\
\mathbf{u}_{n}^{1}\left(T_{n-1}\right) & =\mathbf{C}_{n}^{1}\left(T_{n-1}\right)=\sum_{j=1}^{n} \mathbf{w}_{j}^{1}\left(T_{n-1}\right) .
\end{aligned}
$$

Comparing this with (1.5) and using the uniqueness of the solution for the nonlinear problem, we deduce that $\mathbf{u}_{n}^{1}(t)=\widetilde{\mathbf{u}}_{n}^{1}(t)$ on $\left[T_{n-1}, T_{n}\right]$ for all $n \geq 1$.

Assuming now that for all $n \geq 1$ and a given $k$ we have $\mathbf{C}_{n}^{k}(t)=\sum_{j=1}^{n} \mathbf{w}_{j}^{k}(t)$, $\mathbf{u}_{n}^{k}(t)=\widetilde{\mathbf{u}}_{n}^{k}(t)$ on $\left[T_{n-1}, T_{n}\right]$,we need to show that this also holds for $k+1$. To do so, we prove by recurrence with respect to $n$ that $\mathbf{C}_{n}^{k+1}(t)=\sum_{j=1}^{n} \mathbf{w}_{j}^{k+1}(t)$ on $\left[T_{n-1}, T_{n}\right]$. For $n=1$, we have that $\mathbf{C}_{1}^{k+1}\left(T_{0}\right)=\mathbf{u}_{0}=\mathbf{w}_{1}^{k+1}\left(T_{0}\right)$ and, since $\mathbf{C}_{1}^{k+1}$ and $\mathbf{w}_{1}^{k+1}$ satisfy the same equation and the same initial condition, we conclude that $\mathbf{C}_{1}^{k+1}=\mathbf{w}_{1}^{k+1}$ on $\left[T_{0}, T_{1}\right]$. Next, we suppose that $\mathbf{C}_{n}^{k+1}(t)=\sum_{j=1}^{n} \mathbf{w}_{j}^{k+1}(t)$ on $\left[T_{n-1}, T_{n}\right]$ and prove that $\mathbf{C}_{n+1}^{k+1}(t)=\sum_{j=1}^{n+1} \mathbf{w}_{j}^{k+1}(t)$ on $\left[T_{n}, T_{n+1}\right]$. By checking the initial condition of $\mathbf{C}_{n+1}^{k+1}$ at $T_{n}$ and using the recurrence hypothesis, we find

$$
\mathbf{C}_{n+1}^{k+1}\left(T_{n}\right)=\mathbf{C}_{n}^{k+1}\left(T_{n}\right)+\mathbf{u}_{n}^{k}\left(T_{n}\right)-\sum_{j=1}^{n} \mathbf{w}_{j}^{k}\left(T_{n}\right)=\mathbf{C}_{n}^{k+1}\left(T_{n}\right)+\mathbf{w}_{n+1}^{k+1}\left(T_{n}\right)=\sum_{j=1}^{n+1} \mathbf{w}_{j}^{k+1}\left(T_{n}\right) .
$$

Since $\mathbf{C}_{n+1}^{k+1}$ and $\sum_{j=1}^{n+1} \mathbf{w}_{j}^{k+1}$ solve the same linear problem on $\left[T_{n}, T_{n+1}\right]$ and satisfy the same initial condition at $T_{n}$, we obtain $\mathbf{C}_{n+1}^{k+1}=\sum_{j=1}^{n+1} \mathbf{w}_{j}^{k+1}$ on $\left[T_{n}, T_{n+1}\right]$. Further, for $n \geq 1$ we have

$$
\begin{aligned}
\left(\widetilde{\mathbf{u}}_{n}^{k+1}\right)^{\prime}(t) & =A \widetilde{\mathbf{u}}_{n}^{k+1}(t)+B\left(\widetilde{\mathbf{u}}_{n}^{k+1}(t)\right)+\mathbf{g}(t), \quad t \in\left[T_{n-1}, T_{n}\right], \\
\widetilde{\mathbf{u}}_{n}^{k+1}\left(T_{n-1}\right) & =\mathbf{C}_{n}^{k+1}\left(T_{n-1}\right)=\sum_{j=1}^{n} \mathbf{w}_{j}^{k+1}\left(T_{n-1}\right) .
\end{aligned}
$$

Thus, $\widetilde{\mathbf{u}}_{n}^{k+1}$ and $\mathbf{u}_{n}^{k+1}$ solve the same equation with identical initial condition on $\left[T_{n-1}, T_{n}\right]$ and hence $\widetilde{\mathbf{u}}_{n}^{k+1}=\mathbf{u}_{n}^{k+1}$ on $\left[T_{n-1}, T_{n}\right]$.

The following theorem is essentially a reformulation of Lemma 1 in the usual notation of the parareal algorithm in terms of a coarse and a fine integrator [11].

Theorem 2. Let the coarse propagator $G\left(T_{n}, T_{n-1}, \mathbf{U}\right)$ solve the linear problem

$$
\mathbf{u}^{\prime}(t)=A \mathbf{u}(t) \text { on }\left[T_{n-1}, T_{n}\right], \quad \mathbf{u}\left(T_{n-1}\right)=\mathbf{U},
$$

and let the fine propagator $F\left(T_{n}, T_{n-1}, \mathbf{U}\right)$ solve the nonlinear problem

$$
\mathbf{u}^{\prime}(t)=A \mathbf{u}(t)+B(\mathbf{u}(t))+\mathbf{g}(t) \text { on }\left[T_{n-1}, T_{n}\right], \quad \mathbf{u}\left(T_{n-1}\right)=\mathbf{U} .
$$

Then the solution $\mathbf{u}^{k}$ computed by the nonlinear ParaExp algorithm (1.4)-(1.6) coincides at each time point $T_{n}$ with the solution $\mathbf{U}_{n}^{k}$ computed by the parareal algorithm

$$
\mathbf{U}_{n}^{k}=F\left(T_{n}, T_{n-1}, \mathbf{U}_{n-1}^{k-1}\right)+G\left(T_{n}, T_{n-1}, \mathbf{U}_{n-1}^{k}\right)-G\left(T_{n}, T_{n-1}, \mathbf{U}_{n-1}^{k-1}\right) .
$$


Proof. Using the definition of $\mathbf{u}^{k}$ in (1.6) and the notation of Lemma 1, we have

$$
\begin{aligned}
& \mathbf{u}^{k}\left(T_{n}\right)=\mathbf{u}_{n+1}^{k}\left(T_{n}\right)=\mathbf{C}_{n+1}^{k}\left(T_{n}\right)=\mathbf{C}_{n}^{k}\left(T_{n}\right)+\mathbf{u}_{n}^{k-1}\left(T_{n}\right)-\mathbf{C}_{n}^{k-1}\left(T_{n}\right) \\
= & G\left(T_{n}, T_{n-1}, \mathbf{C}_{n}^{k}\left(T_{n-1}\right)\right)-G\left(T_{n}, T_{n-1}, \mathbf{C}_{n}^{k-1}\left(T_{n-1}\right)\right)+\widetilde{\mathbf{u}}_{n}^{k-1}\left(T_{n}\right) \\
= & G\left(T_{n}, T_{n-1}, \mathbf{C}_{n}^{k}\left(T_{n-1}\right)\right)-G\left(T_{n}, T_{n-1}, \mathbf{C}_{n}^{k-1}\left(T_{n-1}\right)\right)+F\left(T_{n}, T_{n-1}, \mathbf{C}_{n}^{k-1}\left(T_{n-1}\right)\right) .
\end{aligned}
$$

Thus $\mathbf{u}^{k}\left(T_{n}\right)=\mathbf{U}_{n}^{k}$ with $\mathbf{U}_{n}^{k}=\mathbf{C}_{n+1}^{k}\left(T_{n}\right)$.

Theorem 2 shows that the nonlinear ParaExp algorithm is mathematically equivalent to the parareal algorithm (2.2) where the coarse integrator $G$ is an exponential integrator for $\mathbf{w}^{\prime}=A \mathbf{w}$. There is however an important computational difference: due to the linearity of $G$ we can write

$$
\begin{aligned}
& G\left(T_{n}, T_{n-1}, \mathbf{U}_{n-1}^{k+1}\right) \\
= & G\left(T_{n}, T_{n-1}, F\left(T_{n-1}, T_{n-2}, \mathbf{U}_{n-2}^{k}\right)-G\left(T_{n-1}, T_{n-2}, \mathbf{U}_{n-2}^{k}\right)+G\left(T_{n-1}, T_{n-2}, \mathbf{U}_{n-2}^{k+1}\right)\right) \\
= & G\left(T_{n}, T_{n-1}, F\left(T_{n-1}, T_{n-2}, \mathbf{U}_{n-2}^{k}\right)-G\left(T_{n-1}, T_{n-2}, \mathbf{U}_{n-2}^{k}\right)\right)+G\left(T_{n}, T_{n-2}, \mathbf{U}_{n-2}^{k+1}\right),
\end{aligned}
$$

which corresponds to the coarse propagation of a jump over $\left[T_{n-1}, T_{n}\right]$ plus the coarse propagation of $\mathbf{U}_{n-2}^{k+1}$ over a longer time interval $\left[T_{n-2}, T_{n}\right]$. Repeating a similar calculation for $G\left(T_{n}, T_{n-2}, \mathbf{U}_{n-2}^{k+1}\right)$, we derive

$$
\begin{aligned}
G\left(T_{n}, T_{n-2}, \mathbf{U}_{n-2}^{k+1}\right)= & G\left(T_{n}, T_{n-2}, F\left(T_{n-2}, T_{n-3}, \mathbf{U}_{n-3}^{k}\right)-G\left(T_{n-2}, T_{n-3}, \mathbf{U}_{n-3}^{k}\right)\right) \\
& +G\left(T_{n}, T_{n-3}, \mathbf{U}_{n-3}^{k+1}\right),
\end{aligned}
$$

which again corresponds to the coarse propagation of a jump (over two intervals) plus a coarse propagation of $\mathbf{U}_{n-3}^{k+1}$ (over three intervals). This recursion can be repeated, and it will terminate as $\mathbf{U}_{n-n}^{k+1}=\mathbf{U}_{0}$ is known, leading to an alternative, more compact formulation of the nonlinear ParaExp algorithm:

$$
\begin{gathered}
\text { initialize } \mathbf{U}_{n}^{0}=G\left(T_{n}, T_{0}, \mathbf{U}_{0}\right) \quad \text { for } n=0,1, \ldots, N, \\
\mathbf{U}_{n}^{k+1}=G\left(T_{n}, T_{0}, \mathbf{U}_{0}\right)+\sum_{j=1}^{n} G\left(T_{n}, T_{j}, F\left(T_{j}, T_{j-1}, \mathbf{U}_{j-1}^{k}\right)-G\left(T_{j}, T_{j-1}, \mathbf{U}_{j-1}^{k}\right)\right) .
\end{gathered}
$$

Here the coarse integrator is applied in parallel, which is different from parareal. The price to pay is that the coarse integrations now span multiple overlapping time intervals $\left[T_{j}, T_{n}\right]$. As in the original ParaExp algorithm, these linear homogeneous problems can be solved very efficiently using Krylov methods.

We finally investigate the nonlinear ParaExp algorithm numerically. We solve the nonlinear wave equation $u_{t t}=u_{x x}+\alpha u^{2}$ on the time-space domain $[0,4] \times[-1,1]$ with homogeneous Dirichlet boundary conditions and $u(0, x)=e^{-100 x^{2}}, u^{\prime}(0, x)=0$, where the parameter $\alpha \geq 0$ controls the nonlinear character of the problem. The problem is discretized in space using finite differences with $m=200$ equispaced interior grid points on $[-1,1]$. This gives rise to the ODE 


$$
\left[\begin{array}{l}
\mathbf{u} \\
\mathbf{v}
\end{array}\right]^{\prime}=\left[\begin{array}{ll}
O & I \\
L & O
\end{array}\right]\left[\begin{array}{l}
\mathbf{u} \\
\mathbf{v}
\end{array}\right]+\left[\begin{array}{c}
\mathbf{0} \\
\alpha \mathbf{u}^{2}
\end{array}\right]
$$

where $L=\operatorname{tridiag}(1,-2,1) / h^{2}, h=2 /(m+1)$, and the operation $\mathbf{u}^{2}$ has to be understood entry-wise. We partition the time interval $[0,4]$ into $n=20$ slices of equal length and use as fine integrator MATLAB's ode $15 \mathrm{~s}$ routine with a relative error tolerance of $10^{-6}$. For the linear coarse integration we use MATLAB's expm.

In Figure 1 we show the reference solutions $u(t, x)$ for varying $\alpha \in\{0,2,4,6,8.2\}$ on the left, and on the right the error of the ParaExp solution at each time point $t_{j}$ after $k=1,2, \ldots$ iterations. Here a number of $k=0$ iterations corresponds to the error of the ParaExp initialization with the coarse integrator.

The parameter $\alpha=0$ gives rise to a linear problem. We note that for this case the error of the initialization is of order $\approx 10^{-6}$, and not of order machine precision as one would expect from the exponential integration using expm. This is because our reference solution has been computing via ode $15 \mathrm{~s}$ and is of lower accuracy.

For $\alpha=2$ we solve a mildly nonlinear problem and our nonlinear ParaExp algorithm achieves an error of order $\approx 1 e-6$ over all time slices already after 5

iterations. For $\alpha=4$ it requires 7 iterations to achieve this. As the parameter $\alpha$ increases further, the nonlinear character of the wave equation becomes more pronounced and the nonlinear ParaExp algorithm becomes less efficient. For $\alpha \approx 9$ the solution $u(t, x)$ appears to have a singularity in the time-space domain of interest.

\section{References}

1. M. J. GANDER, 50 years of time parallel time integration, Multiple Shooting and Time Domain Decomposition Methods, Springer, pp. 69-113, 2015.

2. M. J. GANDER AND E. HAIRER, Nonlinear convergence analysis for the parareal algorithm, Domain Decomposition Methods in Science and Engineering XVII, Springer, pp. 45-56, 2007.

3. M. J. GANDER, L. HALPERn, AND F. NATAF, Optimal Schwarz waveform relaxation for the one dimensional wave equation, SIAM J. Numer. Anal., 41:1643-1681, 2003.

4. M. J. GANDER AND L. HALPERN, Absorbing boundary conditions for the wave equation and parallel computing, Math. Comp., 74:153-176, 2004.

5. M. J. GANDER AND S. VANDERWAlle, Analysis of the parareal time-parallel timeintegration method, SIAM J. Sci. Comput., 29(2):556-578, 2007.

6. J. Gopalakrishnan, J. SChÖBERL, AND C. Wintersteiger, Mapped tent pitching schemes for hyperbolic systems, arXiv preprint arXiv:1604.01081, 2016.

7. M. J. GANDER AND S. GÜTTEL, PARAEXP: A parallel integrator for linear initial-value problems, SIAM J. Sci. Comput., 35(2):C123-C142, 2013.

8. M. J. GANDER AND M. PETCU, Analysis of a Krylov subspace enhanced parareal algorithm, ESAIM Proc., 25:45-56, 2008.

9. S. GÜTTEL, A parallel overlapping time-domain decomposition method for ODEs, Domain Decomposition Methods in Science and Engineering XX, Springer, pp. 483-490, 2013.

10. G. Koolj, M. Botchev, AND B. Geurts, A block Krylov subspace implementation of the time-parallel ParaExp method and its extension for nonlinear partial differential equations, $\mathrm{J}$. Comput. Appl. Math., 316:229-246, 2017.

11. J.-L. LIONS, Y. MADAY, AND G. TURINICI, A "parareal" in time discretization of PDE's, C. R. Acad. Sci. Paris Sér. I Math., 332:661-668, 2001. 

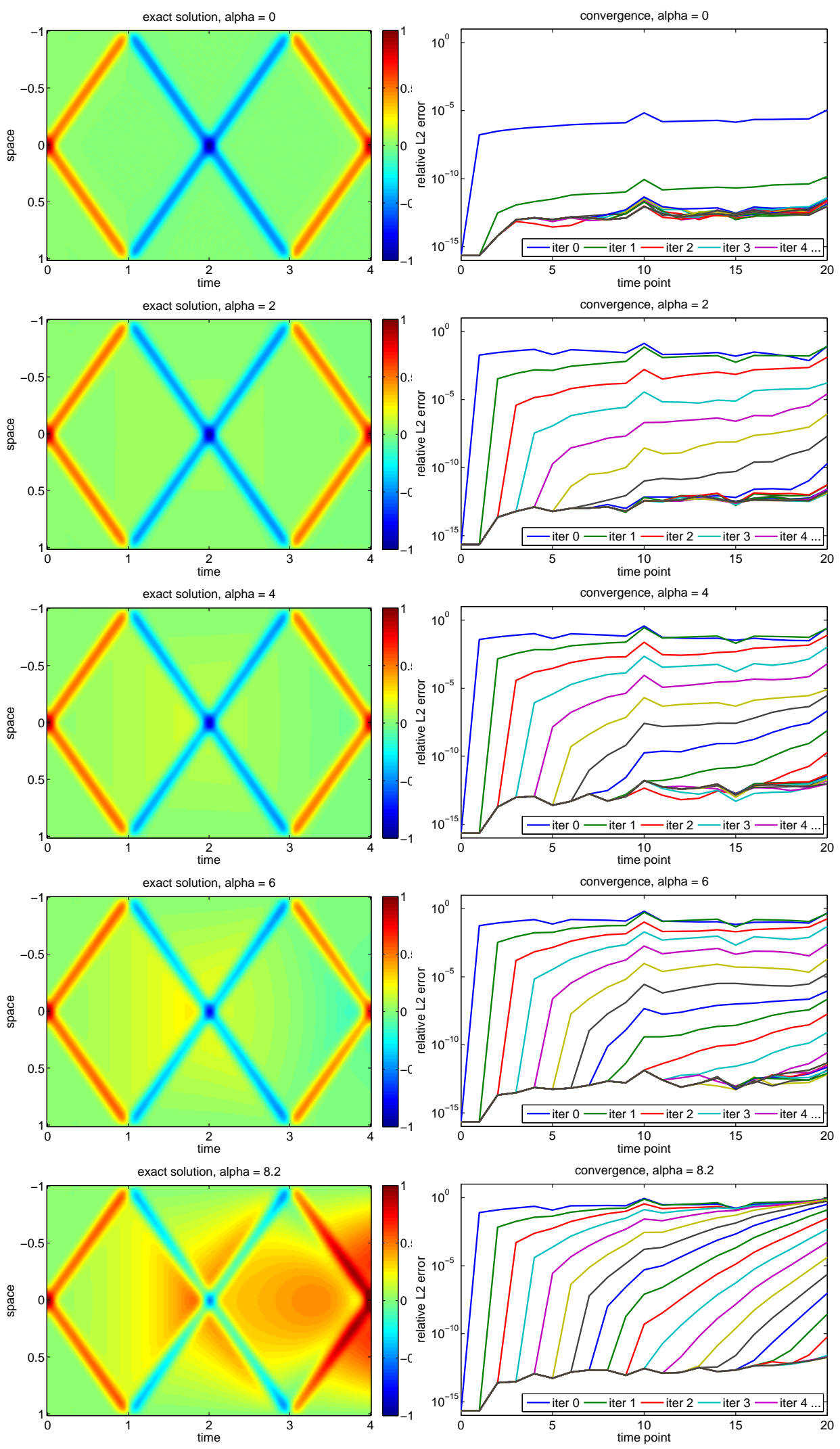

Fig. 1 Exact solutions (left) and convergence (right) of the nonlinear ParaExp algorithm applied to a nonlinear wave equation with varying parameter $\alpha \in\{0,2,4,6,8.2\}$ (top to bottom). 